\title{
DISLOCATION DISTRIBUTIONS IN TWO DIMENSIONS
}

\author{
A. N. Gulluoglu+, D. J. Srolovitz ${ }^{+}$, R. LeSar ${ }^{*}$, and P. S. Lomdahl* \\ +Department of Materials Science and Engineering, University of Michigan \\ Ann Arbor, MI 48109 USA \\ *Theoretical Division, Los Alamos National Laboratory \\ Los Alamos, NM 87545 USA \\ (Received May 1, 1989) \\ (Revised May 22, 1989)
}

\section{Introduction}

Electron microscopy studies of deformed metals have shown that the spatial distribution of dislocations is decidedly non-random. In most cases, the dislocations are arranged into definite patterns. The observed patterns are often in the form of dislocation cells/sub-grains which consist of relatively dislocation free regions surrounded by walls composed of dense tangles of dislocations. The cell size is known to depend sensitively on the conditions present during deformation (i.e. strain rate, temperature, etc.) If the conditions under which a material is being deformed is abruptly changed, transients in the stress/strain curve are observed which persist until the dislocation patterns appropriate to the new conditions are established. This indicates that dislocation patterns play a major role in determining the mechanical response of a material.

The existence of dislocation patterns indicates the importance of dislocation-dislocation interactions. These interactions are of two forms: 1) long-range elastic interactions, and 2) short-range topological interactions (i.e. dislocation tangling). In the present paper, we focus on dislocation pattern formation in the framework of a simulation procedure which accounts for the interactions between a large number of dislocations (typically $10^{3}$ dislocations) at densities which are typical of deformed metals.

Recently, Lepinoux and Kubin (1) and Ghonhiem and Amodeo (2) presented simulation procedures with similar goals. Lepinoux and Kubin (1) described the evolution of dislocation patterns in the framework of a cellular automata model, while Ghonhiem and Amodeo (2) developed a technique based upon the molecular dynamics method. Both studies accounted for dislocation interaction in terms of the elastic interactions between pairs of dislocations which they truncated at a predefined cut-off radius $R_{c}$. In our study, we have employed a molecular dynamics-type simulation method, similar to that employed by Ghonhiem and Amodeo (2), and have examined the influence of the elastic interaction cut-off procedure. The method by which the dislocationdislocation interactions are cut-off is extremely important since the force one dislocation exerts on another decays very slowly $(1 / \mathrm{r})$. This suggests that a cut-off at any finite distance ignores most of the elastic interactions (i.e. the integral of $1 / \mathrm{r}$ from an inner cut-off distance to $R_{c}$ is a poor approximation of the integral to infinity for $R_{C}<\infty$ ). Such truncation of the interaction distance is known to introduce decidedly unphysical effects in simulations of ionic systems, where the forces decay more rapidly than in the present case (3).

In the present paper, we determine the dislocation patterns formed when an initially random distribution of edge dislocations on parallel slip planes relaxes under the influence of their mutual interactions and with no applied stress. In the following section, the details of the simulation procedure employed are described and a new method for avoiding the interaction cut-off issue is discussed. The dislocation patterns for simulations both with and without a cut-off are shown in Section 3 . The dislocation pattern is then analyzed in terms of dislocation pair distribution functions.

\section{Simulation Method}

In the present model, the dislocation lines are assumed to be straight, parallel, and are of edge character. Under these condition, the simulation is essentially two-dimensional. The dislocations can move both along their slip plane and normal to it by glide and climb, respectively. The simulation method can also account for arbitrary externally applied stresses. For simplicity, we restrict our attention to the case where all dislocations have parallel (or anti-parallel) Burgers vectors, the applied stress is zero and the temperature is sufficiently low that climb may be neglected. These simulations, therefore, correspond to low temperature, stage I deformation of single crystals (oriented for single slip). 
The total force on one dislocation due to all of the others may be expressed in terms of its Burgers vector $\left(b_{i}\right)$ and the stress fields of each dislocation in the group $\left(\sigma_{j}\right)$. The Peach-Koehler (4) equation yields such a force per unit length on dislocation $i$, which is given by

$$
\frac{\overrightarrow{F_{i}}}{\mathbf{L}}=\sum_{\mathbf{j} \neq \mathbf{i}}\left(\overrightarrow{\mathrm{b}_{\mathrm{i}}} \cdot \overrightarrow{\sigma_{j}}\right) \times \vec{\xi}
$$

where the summation is over all dislocations excluding dislocation $i, \sigma_{j}$ is evaluated at the position of dislocation $i$, and $\xi$ is the dislocation line direction. By resolving this force parallel to the Burgers vector of dislocation $i$, the glide force is:

$$
\frac{F_{i}^{g}}{L}=\sum_{j \neq i} \frac{\mu b_{i} b_{j}}{2 \pi(1-v)} \frac{x_{i j}\left(x_{i j}^{2}-y_{i j}^{2}\right)}{\left(x_{i j}^{2}+y_{i j}^{2}\right)^{2}}
$$

where we have chosen the $\mathrm{x}$-direction to lie parallel to the Burgers vector and $\mathrm{x}_{\mathrm{ij}}$ and $\mathrm{y}_{\mathrm{ij}}$ are the components of the relative separation between dislocations $\mathrm{i}$ and $\mathrm{j}$.

The sums in Eq. (2) are difficult to evaluate due to the long-range interactions between dislocations, which decay as the inverse of the interdislocation distance. Two alternative approaches are considered: 1) truncation of interactions at some cut-off distance $R_{c}$ or 2 ) infinitely repeating the simulation cell so as to fill all space and summing the interactions due to all of the replicas. Employing the second option, the force on a dislocation, 1, due to another dislocation, 2, and its replicas in the direction normal to the Burgers vector can be summed simply by noting that this arrangement is equivalent to a low angle grain boundary whose stress field can be determined analytically (5). Since the force on dislocation 1 due to the low angle boundary corresponding to dislocation 2 decays very quickly away from the low angle boundary (i.e. nearly exponentially), only a few dislocation 2 boundaries (spaced 1 unit cell apart in the x-direction) must be included to account for the remainder of the twodimensional lattice of dislocation 2 replicas. The extra computational burden associated with explicitly performing this sum is avoided by constructing a two-dimensional table of forces on one dislocation due to another dislocation (and all of its replicas) and interpolating. This method for calculating the force guarantees that all dislocation interactions out to infinite distance are incorporated and no interaction cut-off distance, $R_{c}$, is employed. However, solely for the sake of comparison with simulation methods which employ an explicitly interaction cutoff distance, a small number of calculations were performed in which a cut-off distance was used $\left(R_{c}=0.5\right.$ cell length).

In the present simulations, the magnitudes of the constants in Eq. (2) were chosen to represent copper $\left(\mu=5.5 \times 10^{10} \mathrm{~N} / \mathrm{m}^{2}, v=0.324,|\mathrm{~b}|=2.5 \times 10^{-10} \mathrm{~m}\right)$. The computational cell was a square with edge length $10^{-6} \mathrm{~m}$ and the dislocation density was fixed at $10^{15} \mathrm{~m}^{-2}$, corresponding to $10^{3}$ dislocations. Periodic boundary conditions were assumed, implying that a dislocation that moves past the right edge of the cell reappears at the left edge. The starting configuration was obtained by randomly placing edge dislocations in the computational cell with an equal density of parallel and anti-parallel Burgers vectors. The equilibrium dislocation patterns were obtained by moving the dislocations to positions where the total glide force on each dislocation was zero (i.e. the dislocations were not allowed to climb). This was accomplished by employing a large frictional force on the motion of each dislocation within the framework of our Molecular Dynamics procedure. In order to guarantee that the dislocations were at minimum energy positions, the Peierls stress was set to zero in the present calculations.

The equilibrium (no climb) configuration of the dislocations is non-random due to the dislocationdislocation interactions. The relative arrangements of the dislocations can be characterized in terms of dislocation pair distribution functions. Since the stress field (and hence the interaction force) of an edge dislocation is anisotropic, two-dimensional distribution functions are most appropriate for analyzing the dislocation patterns. We define the two-dimensional pair distribution function $F(x, y)$ as the difference in the local density of like $\left(f^{+}\right)$ signed (i.e., parallel Burgers vector) and unlike $\left(f^{-}\right)$signed dislocations as a function of their relarive position $(x, y), F(x, y)=\left[f^{+}(x, y)-f^{-}(x, y)\right] / \rho$, where $\rho$ is the mean dislocation density. $f^{+}(x, y) d x d y$ is calculated as the number of edge dislocations, with the same Burgers vector as the dislocation at the origin, that fall into the grid element $(x \rightarrow x+d x, y \rightarrow y+d y)$ normalized by the dislocation density times the area of that grid element and averaged over all possible dislocations as origins. The distribution functions displayed in the next section have been averaged over the relaxed configurations from 8 simulations corresponding to a total of 8,000 dislocations. 


\section{Results}

Dislocation patterns found using the relaxation method described for the infinitely repeated cell (i.e. no cutoff) are displayed in Fig. la. In this figure, each dislocation is represented by either $\perp$ or T, depending on the sign of the Burgers vector. While a well ordered structure is not observed, short-range order is clearly present. The two main features observed in the microstructure are: 1) the presence of dislocation dipoles and 2) dislocation walls consisting of vertically aligned edge dislocations with the same Burgers vector. A significant fraction of the dislocations have formed very closely spaced dipoles which are extremely stable in the absence of climb. The dislocation walls which are observed are similar to that seen in low angle grain boundaries. However, these walls are of only finite extent and are not nearly as narrow as would be expected of true low angle grain boundaries. An additional, albeit less pronounced feature, of the dislocation pattern observed is the presence of dislocation walls lying at $45^{\circ}$. Unlike the vertical walls, however, these walls appear to consist of equal numbers of dislocations with positive and negative Burgers vectors. No particular order is noticed in the direction parallel to the Burgers vector (i.e. the $x$-direction).

For the sake of comparison, additional simulations were performed without the infinitely repeated cell and with an interaction range cut-off $\left(R_{c}\right)$ equal to half the cell size. Figure $1 b$ shows the dislocation pattern observed under such conditions shortly before complete relaxation. The completely relaxed configuration is very similar to the one showed in Fig. 1b, but with dislocation walls so sharp that the individual dislocations could no longer be distinguished. Under these conditions, the dislocation walls extend over the whole vertical dimension of the simulation cell and contain dislocations which are almost entirely of the same sign. Four dislocation walls are observed and the sign of the Burgers vector alternates from wall to wall. This is very similar to a polygonized structure. Further calculations with different values of $R_{c}$, however, show that the spacing between walls changes with the value of $R_{c}$. In particular, it appears that the system tries to adjust the interwall spacing to be equal to $\mathrm{R}_{\mathrm{d}} / 2$.

Since the dependence of the interwall spacing on $R_{c}$ makes the simulations with finite interaction distance cut-off suspect, only those simulations performed using the infinitely repeated cell (no cut-off) method (e.g. Fig. 1a) were further analyzed. The two-dimensional dislocation pair distribution function is $F(x, y)$ is shown in $F i g$. 2 , where the dark regions indicate high densities of dislocations with the same sign as that at the origin and the white regions indicate high densities of oppositely-signed dislocations. This distribution function shows a strong attraction between unlike dislocations at small $x$, particularly at $45^{\circ}$ (the $45^{\circ}$ line corresponds to the equilibrium positions of a pair of anti-parallel edge dislocations). (The extension of this region along the $y$-axis is attributable to the :mall but finite grid size used in creating the contour plot.) There is a strong propensity for like-signed disloca ions along the $y$-axis, consistent with our observations of dislocation walls in the microstructure, Fig. $1 \mathrm{a}$. The strong preference for like-signed dislocations along the y-axis is balanced by parallel, nearby regions of oppositely-signed dislocations. Along the $x$-axis, the net dislocation density appears to show some oscillation, going from near zero to reasonably large densities of like-signed dislocations.

\section{Discussion and Conclusions}

When a dislocation interaction cut-off distance is employed, oppositely-signed dislocations are pushed outside the cut-off range leading to a buildup of sharp like-signed dislocation walls at the cut-off distance. The formation of such walls prevents the formation of low-energy dislocation dipoles. In the present calculations, this artefact has been removed by the introduction of an infinitely repeated unit cell with no interaction cut-off. Similar observations have been made in the simulation of ionic fluids (3) and have been overcome by the introduction of an Ewald summation method or by properly employing a screened long-distance interaction formalism.

The dislocation microstructures observed in the infinitely repeated simulation cell calculations demonstrate that equilibrated dislocation spatial distributions are correlated. Both the microstructures and pair distribution functions show that dislocation walls and dislocation dipoles constitute the preferred low-energy structures. The separation between the dislocations in the dipoles are limited to spacings of order the mean interdislocation separations (i.e. 0.03 on the length scales of Figs. 1 and 2). The dislocation wall structures, on the other hand, extend over distances of order ten times the mean interdislocation spacing. The oscillations in the dislocation density in the direction parallel to the Burgers vector are rather weak and are much more easily observed in the dislocation pair distribution function plots than in the microstructures. The wavelength of this oscillation is of order the mean interdislocation spacing. However, due to our limited statistics, it is not possible to determine if this oscillatory behavior is real or purely statistical. Assuming the dislocation at the origin is positive, nearby negative dislocations are attracted in toward the origin leaving a net increase in positive dislocations behind. This positive region attracts its own cloud of negative dislocations and hence the oscillations continue away from the origin, albeit with decreasing amplitude. 


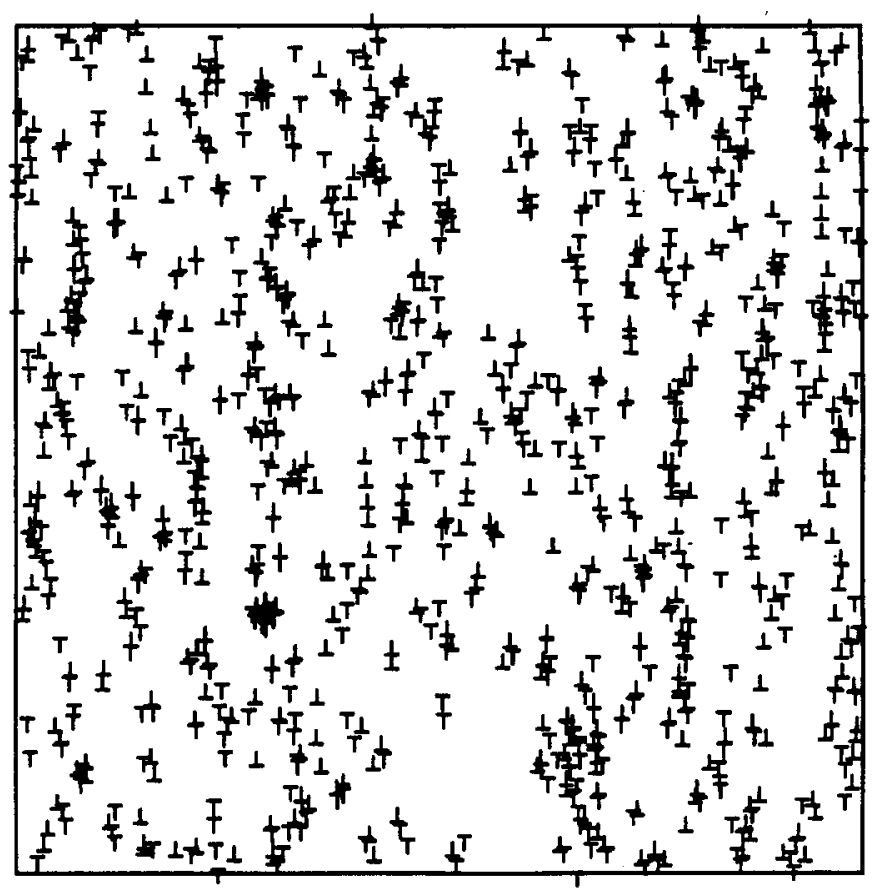

(a)

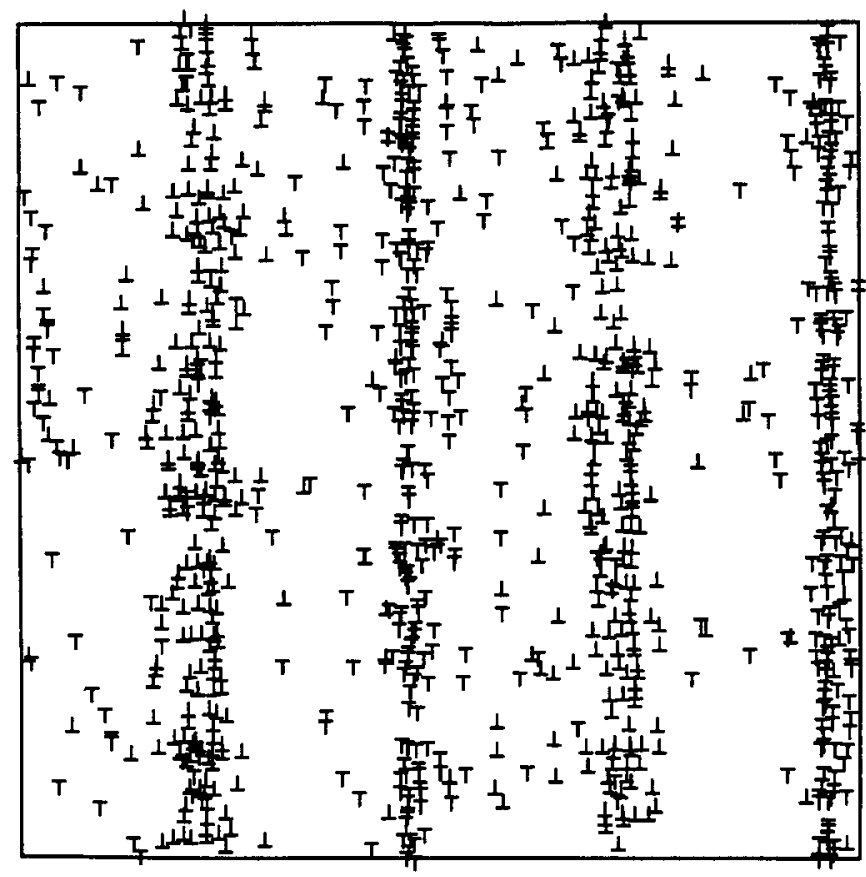

(b)

FIG. 1 Simulated dislocation microstructures with a dislocation density of $10^{15} \mathrm{~m}^{-2}$ corresponding to $10^{3}$ edge dislocations in a $1 \mu \mathrm{m} \times 1 \mu \mathrm{m}$ simulation cell using (a) the infinitely repeated simulation cell (i.e. no cut-off) and (b) the truncated interaction distance $\left(R_{c}=0.5\right.$ cell size) methods. 
In order to determine the effects of dislocation density, simulations were performed for $\rho=10^{11}, 10^{13}$, and $10^{15} \mathrm{~m}^{-2}$. The dislocation pair distribution functions $F(x, y)$ for these three densities yield nearly identical structures when the $x$ and $y$ axes are scaled by the mean interdislocation spacing, $\rho^{-1 / 2}$. This result is expected since there is only one length scale in this problem, i.e $\rho^{-1 / 2}$.

Since the pair distribution function indicates the presence of dislocation walls of finite extent and a periodicity in the direction perpendicular to these walls, we may view this structure as consisting of ill-formed dislocation cells. Within this interpretation of the microstructure, the observed scaling of all lengths by the dislocation density implies that the dislocation cell size varies with the mean dislocation density as $\rho^{-1 / 2}$. Such a scaling has been predicted on theoretical grounds by Holt (6) based upon a spinodal decomposition-like theory.

Comparison of the present dislocation patterns with those observed in deformed metals is difficult since real dislocation cell structure depends sensitively on the deformation conditions (e.g. strain rate, temperature). However, careful etch-pit studies of dislocation patterns in silver single crystals have been performed by Worzala and Robinson (7). The micrographs obtained using etch pitting show a remarkably strong correspondence with our simulated microstructures. Both the simulated and silver micrographs show the presence of a rough cell structure consisting of both dislocation clusters and crude dislocation walls. The applied stress driving deformation will sharpen the cell structure by overcoming local barriers to dislocation motion. Additionally, application of an external stress effectively cuts off the dislocation interaction at a finite distance. This can be most easily seen by examining the case of dislocation dipoles, where the application of an applied stress reduces the maximum separation between bound dipoles from infinity at $\sigma=0$ to a distance which decays as $1 / \sigma$ for finite $\sigma(8)$. [Additionally, the presence of additional dislocations tend to destabilize dipoles (9).] Therefore, the application of an external stress results in a stress-dependent dislocation interaction range cut-off. Our finite cut-off distance results (Fig. 1b) show that this leads to the formation of much sharper dislocation walls.

Since dislocation cell structure is sensitive to deformation conditions, future work in this area will focus on the effects of applied stress, climb (i.e. temperature), and obstacles on dislocation structure, its evolution and the plastic properties of crystals.

\section{References}

1. J. Lepinoux and L. P. Kubin, Scripta Metall. 21, 833 (1987).

2. N. M. Ghonhiem and R. Amodeo, in "Nonlinear Phenomena in Materials Science," ed. by G. Morton and L. P. Kubin, Trans. Tech. Publications, Aedermannsdorf, Switzerland (1988).

3. J. P. Hansen, "Molecular Dynamics Simulation of Statistical-Mechanical Systems," ed. by G. Ciccotti and W. G. Hoover, North-Holland, Amsterdam (1986).

4. M. Peach and J. S. Koehler, Phys. Rev. 80, 436 (1950)

5. A. H. Cottrell, "Dislocations and Plastic Flow in Crystals," Oxford University Press, Fair Lawn, N.J., 1953.

6. D. L. Holt, J. Appl. Phys. 41, 3197 (1970).

7. F. J. Worzala and W. H. Robinson, Phil. Mag. 15, 939 (1967).

8. R. Eykholt and D. J. Srolovitz, J. Appl. Phys., in press.

9. P. D. Neumann, Acta Metall. 19, 1233 (1971). 


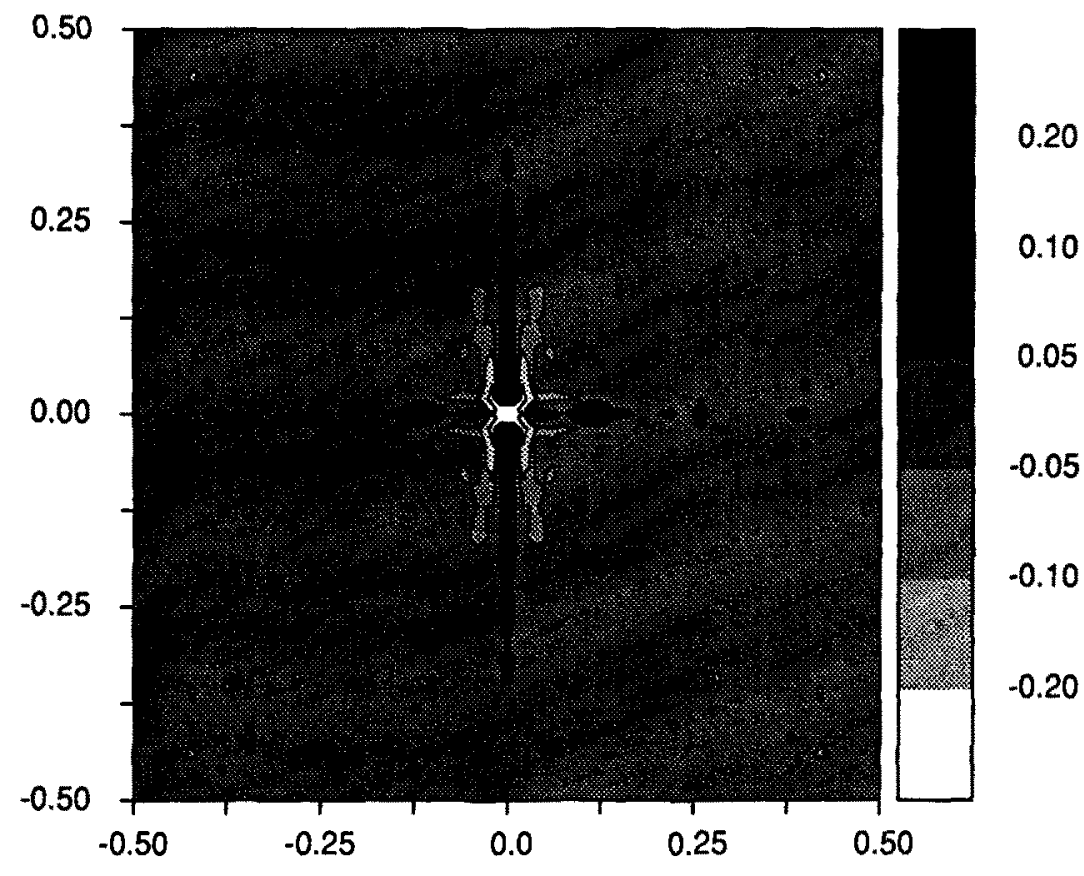

(a)

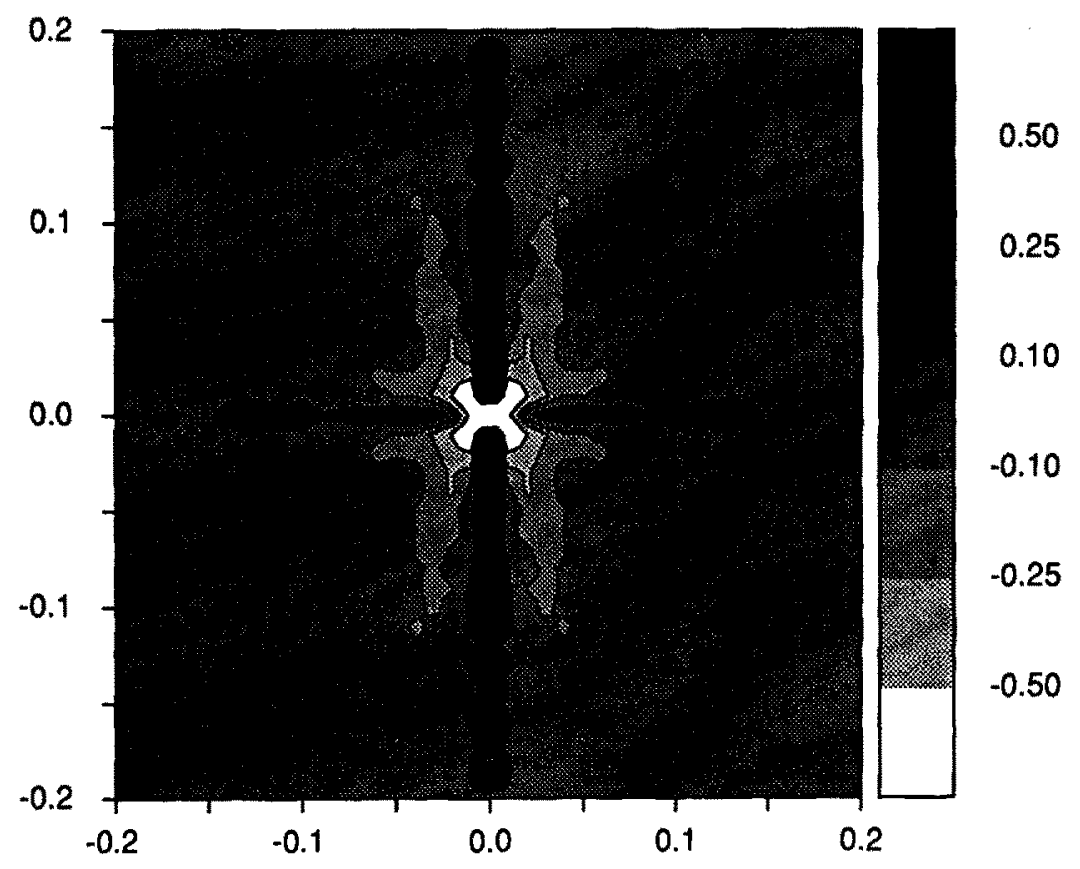

FIG. 2 Contour plots of the dislocation pair distribution function $F(x, y)$ averaged over 8 simulations. Figure $b$ was produced with twice the resolution of Fig. $a$. The $x$ and $y$ axes have been scaled by the simulation cell size. The light (dark) regions correspond to high concentrations of dislocations with Burgers vectors opposite to (same as) the dislocation at the origin. The distribution function was determined in one quadrant and reflected. 